\title{
NUMERICAL MEAN ELEMENTS FOR ASTEROID ORBITS
}

\author{
ANNE LEMAITRE \\ Département de Mathematique - FUNDP \\ 8 Rempart de la Vierge B5000 Namur Belgique \\ E-mail: alemaitr@math.fundp.ac.be
}

\section{Introduction}

In the context of the search of asteroid families (i.e. identification of minor planets as potential fragments of an old bigger body), the calculation of proper elements plays an important role. They are quasi-invariants of the motion, obtained by a double averaging process of the restricted $\mathrm{N}$-body problem; firstly the osculating elements are averaged over the short periodic terms (namely the longitudes of the asteroid and of the perturbing planets) so to get the mean elements, and secondly, the mean elements are averaged over the long periodic terms (longitudes of the pericenters and of the nodes of the asteroid and of the perturbing planets) to obtain the proper elements.

The calculation of proper elements for asteroid orbits presently refers to two different computations; a powerful analytical theory, developed by Milani and Knežević $(1990,1992,1994)$ and a semi-numerical approach due to Lemaitre and Morbidelli (1994). The analytical theory, based on series expansions in eccentricity and inclination, is particularly suitable for low inclination and low eccentricity orbits, while the semi-numerical method is convenient for the orbits with either large eccentricities or large inclinations. The overlapping region has been tested and is situated about $17^{\circ}$ in inclination (see Knežević et al 1994).

Up to now even the semi-numerical approach has used the analytical mean elements as initial conditions for the computation of the proper elements; those mean elements, also obtained by series expansions by Milani and Knežević, could be sources of errors in the calculation of the proper ones for highly inclined or eccentric orbits.

The purpose of this paper is to compare, at least for the numbered asteroids, the analytical mean elements with a corresponding set of numerical 
mean ones. For significative differences in the results, proper elements are then recalculated with the numerical initial conditions and compared to their previous values.

\section{Numerical integration}

We perform a numerical integration by ORBIT8V (implemented by Milani) using the digital filtering techniques (Carpino et al 1987) to average over the short periodic terms. Because of the presence of high eccentricities and high inclinations in our data set, the fixed step integrator has been replaced by a variable step integrator, namely RA15 (Everhart 1985). The integration of 6158 asteroids (1994 catalogue) covers 2000 years and takes into account the perturbations of Jupiter, Saturn, Uranus and Neptune on the motion of the minor planet. Outputs are listed every 100 years and by choice of a filter $n^{\circ} 100$, the filtered data, corresponding to the elimination of the periods between 2 and 200 years, are available between 600 and 1400 years. The reference epoch has been arbitrarily chosen at 1000 years.

To test the validity of the averaging process a triple index $\left(p_{a}, p_{e}\right.$ and $p_{i}$ ) is given for each planet, corresponding to the RMS values of a constant fit of the filtered numerical values of the semi-major axis $a$ and of quadratic fits for the filtered values of the eccentricity $e$ and sine of the inclination $\sin i$. Let us remind that the elimination of the longitudes in the Hamiltonian function theoretically corresponds to a constant Delaunay momentum $L=\sqrt{\mu a}$, which leads to a constant value of $a$; the mean Hamiltonian so obtained should contain, as main terms in a series expansion, a quadratic term in $e$ and in $i$, which is checked by two quadratic fits for those elements. The RMS is given on a logarithm scale with respect to $10^{-5}$; for example, $p_{a}=-1$ means that the RMS value of $a$ with respect to a constant is about $10^{-6}$ and $p_{e}=+2$ means that the RMS value of $e$ with respect of a quadratic function is about $10^{-3}$. This allows the user to extract several subsets from the main data set, by changing the precision of each of the three mean elements. Results about the triple index are given in table 1

\section{Comparisons with analytical mean elements}

With the result of the numerical integration given at the epoch 1000 as initial condition, an analytical mean element has been calculated by means of SHORT6.3 software. As well as for the numerical mean elements a quality code is given (Milani and Knežević 1994), indicating special situations such as closeness to the mean motion resonances or extremal values of the semimajor axis. 4263 asteroids have a quality code 0 ; combining the two criteria ("good" triple index and "good" analytical quality code) 4023 planets are retained for the comparison. 

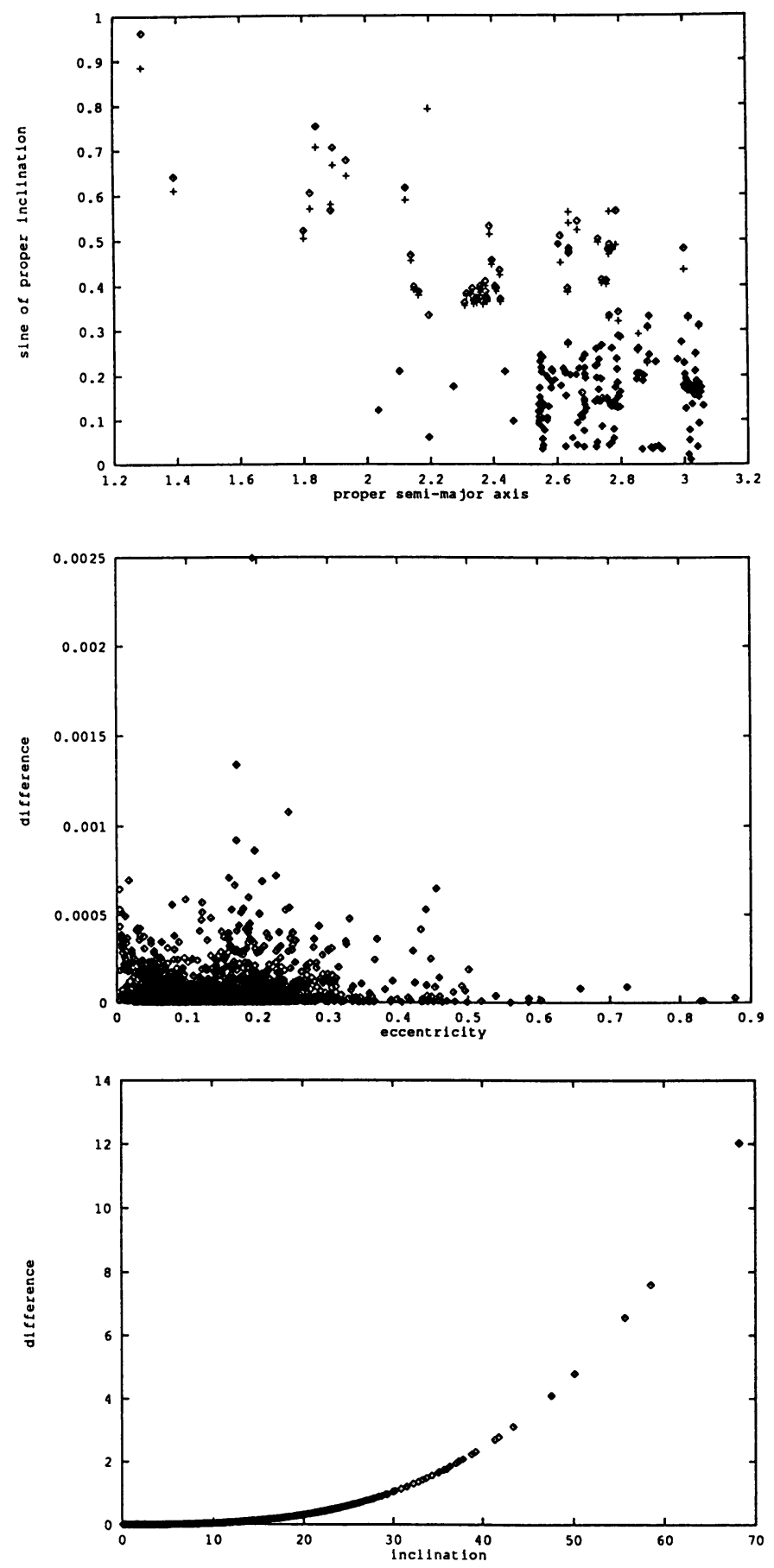

Figure 1. Differences in semi-major axis, eccentricity and inclination (in degrees) for comparable (good quality codes for both elements) analytical and numerical mean elements (4023 planets are plotted) 
TABLE 1. Number of asteroids corresponding to a given value of the index, respectively for $a, e$ and $i$; for the extremal values, the asteroid is identified

\begin{tabular}{|c|ccccccccc|}
\hline Element & -4 & -3 & -2 & -1 & 0 & 1 & 2 & 3 & 4 \\
\hline$a$ & 1 & 11 & 127 & 807 & 4200 & 754 & 163 & 91 & $\begin{array}{c}4 \\
(2340)\end{array}$ \\
$e$ & 0 & 0 & 4 & 259 & 5001 & 563 & 256 & 75 & $\begin{array}{c}(944,5145) \\
(2100,2102)\end{array}$ \\
$\sin i$ & 0 & 0 & $\begin{array}{c}(4034,4232) \\
103\end{array}$ & 1518 & 4038 & 458 & 40 & 1 & 0 \\
\hline
\end{tabular}

The figures 1 give the differences observed in semi-major axis, eccentricity and inclination (in degrees) between the two sets of mean elements; it is obvious that the differences in $a$ and $e$ are much smaller than in $i$, where the curve is a quadratic function of the inclination. Even in the interval between $15^{\circ}$ and $30^{\circ}$ the differences exceed $1^{\circ}$ i.e. $10^{-2}$ for $\sin i$. This result confirms the now classical result obtained by Kozai (1962) who showed the difference of topology between the mean phase space in the circular restricted problem between low and high inclinations; the analytical mean phase space does not take this peculiar topology (with apparition of a critical curve) into account (see Lemaitre and Morbidelli 1994 for a larger discussion).

\section{Consequences for proper elements}

Out of the 4023 planets, 279 have been isolated, showing either differences in $a$ larger than $10^{-4}$, or differences in $e$ larger than $510^{-4}$ or differences in $i$ larger than $1^{\circ}$. Corresponding proper elements have been calculated for both sets of mean elements and the results are plotted in figure 2 for the inclinations and in figure 3 for the frequencies.

A systematic shift in the frequency space as well as in the $(a, i)$ space can be noticed; it means that for family determination the two sets of proper elements are equivalent, because only the mutual distances between the planets are important; for other purposes, it is obvious that differences of a few arcseconds in the frequencies could be very important, in particular for the location or the proximity of the secular resonances. 


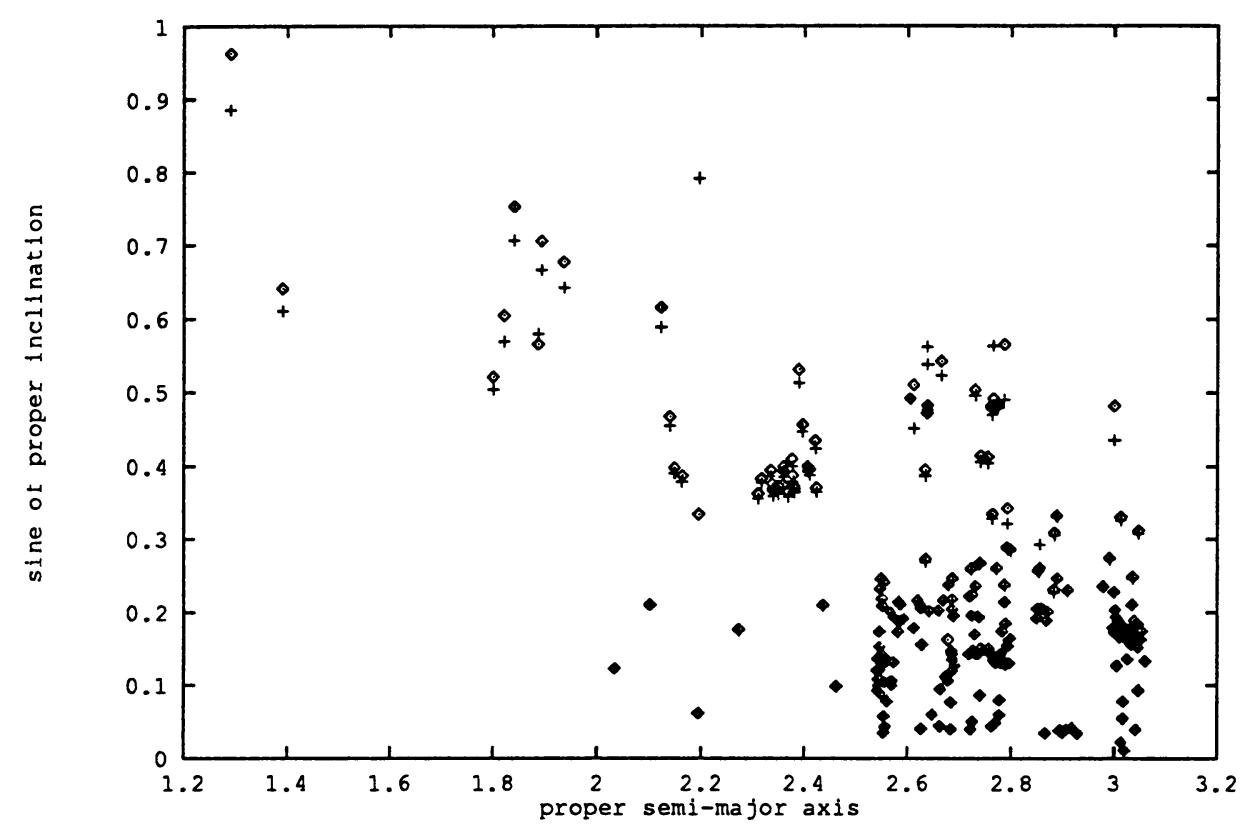

Figure 2. Differences between the proper inclinations calculated from analytical mean elements $(+)$ and the proper inclinations calculated from numerical mean elements $(\diamond)$ for 279 planets

\section{Conclusions}

For inclinations lower than $15^{\circ}$, analytical mean elements are equivalent to the numerical ones whatever the semi-major axis or the eccentricity could be; for mean inclinations between $15^{\circ}$ and $30^{\circ}$, differences are of the order of $1^{\circ}$; this difference is reported in proper inclination in a linear way, which allows the user to predict the difference in proper elements, knowing the difference in mean elements. For inclinations higher than $30^{\circ}$, numerical mean elements should be used, but in a separate file, the worst thing being to mix up two sets of data. If the reader is interested in some result of the present paper, please contact the author by Email or check the public domain database ftp.dm.unipi.it, where osculating, mean and proper elements are available.

\section{Acknowledgements}

I would like to thank Andrea Milani for useful comments and discussions about this contribution, as well as for "public domain" software ORBIT8V which I have intensively used for these results. 


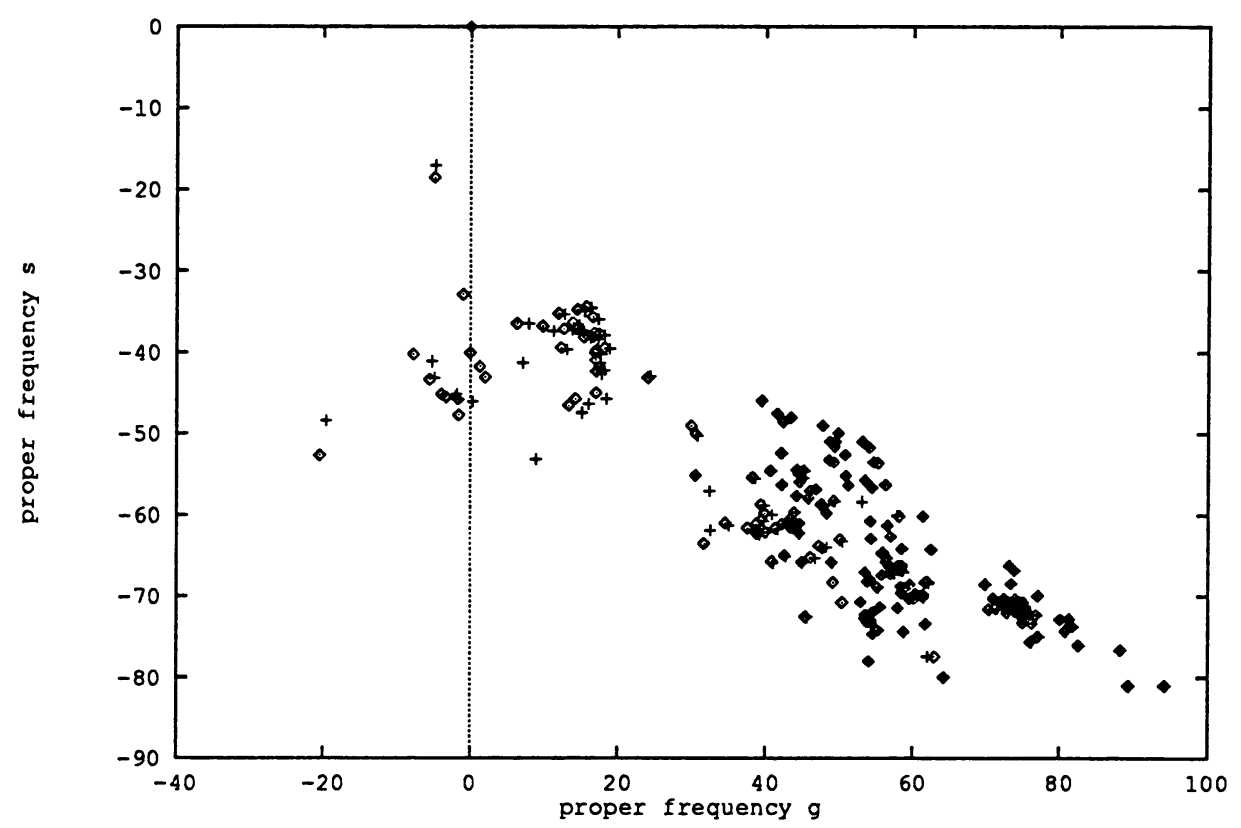

Figure 3. Proper frequencies $g$ and $s$, given in arcseconds per year, calculated from analytical mean elements $(t)$ and the proper frequencies calculated from numerical mean elements $(\diamond)$ for 279 planets

\section{References}

Carpino, M., Milani, A., Nobili, A.: 1987, "Long term numerical integrations and synthetic theories for the motion of the outer planets", Astron. Astroph., 181, 182-194

Everhart, E.: 1985, "An efficient integrator that uses Gauss-Radau spacings", Dynamics of Comets: their origin and evolution, Eds A. Carusi and G.B. Valsecchi, D. Reidel, Dordrecht, 185-202

Knežević Z., Froeschlé Ch., Lemaitre A., Milani A., Morbidelli A.: 1994, "Comparison of two theories of asteroid proper elements", Astron. Astroph., 293, 605-612

Kozai, Y.: 1962, "Secular Perturbations of asteroids with high inclinations and eccentricities", Astron. J., 67, 591-598

Lemaitre A., Morbidelli A.: 1994, "Calculation of Proper Elements for high inclined asteroidal orbits", Celest. Mech., 60, 29-56

Milani, A., Knežević, Z.: 1990, "Secular Perturbation Theory and Calculation of Asteroid Proper Elements", Celest. Mech. and Dyn. Astr., 49, 347-411

Milani, A., Knežević, Z.: 1992, “Asteroid proper elements and secular resonances”, Icarus, 98, 211-232

Milani, A., Knežević, Z.: 1994, "Asteroid proper elements and the dynamical structure of the asteroid belt", Icarus, 107, 219-254 